\title{
COST DRIVERS: REFLEXÕES SOBRE DEFINIÇÕES, CONCEITOS, UTILIDADES E APLICABILIDADES
}

\section{COST DRIVERS: REFLECTIONS ON DEFINITIONS, CONCEPTS, UTILITIES AND APPLICABILITY}

0 artigo foi aprovado e apresentado no XXII Congresso Brasileiro de Custos, realizado de 11/11 a 13/11 de 2015, em Foz do Iguaçu (PR)

\section{RESUMO}

Este estudo apresenta uma análise crítica das definições e dos conceitos de cost drivers, considerando os aspectos utilitaristas e de aplicabilidade, bem como os frameworks do Activity-Based Costing (ABC) e da Gestão Estratégica de Custos (GEC) que utilizam a expressão cost drivers, conforme exposta na literatura brasileira e internacional. Para isso, foi verificado nos materiais bibliográficos disponíveis, os conceitos de cost drivers em sentido amplo, com o significado de determinantes de custos e com a denotação de direcionadores de custos. O estudo evidencia os vieses existentes na literatura brasileira no uso dos conceitos e contribui, por meio de críticas, às definições e conceitos, apresentando uma proposta conceitual para os cost drivers, segundo suas características. O trabalho revela a essência dos determinantes de custos como fatores estratégicos e os direcionadores de custos como elementos distribuidores de montantes de custos. Para os gestores, estes proveem maior acurácia na informação para a mensuração do desempenho operacional, ao passo que aqueles definem rumos estratégicos. Para a gestão, essa diferença primordial pode afetar a maneira como ocorre o processo decisório.

Palavras-chave: Cost Drivers; Determinantes de Custos; Direcionadores de Custos; ABC; GEC.

\begin{abstract}
This paper presents a critical analysis of the definitions and concepts of cost drivers, considering the utilitarian and applicability aspects, as well as the Activity-Based Costing (ABC) and Strategic Cost Management (GEC) frameworks that use the expression cost drivers, as exposed in the Brazilian and international literature. For this, it was verified in the available bibliographic materials, the concepts of cost drivers in a broad sense, with the meaning of cost determinants and $A B C$ cost drivers concepts. The study highlights the biases existing in the Brazilian literature on the use of concepts and contributes, through criticism, to the definitions and concepts, presenting a conceptual proposal for both cost drivers, according to their characteristics. The paper reveals the essence of cost determinants as strategic factors and cost drivers as elements of cost amounts. For managers, these provide greater accuracy in information for measuring operational performance, while those define strategic directions. For management, this primary difference can affect the way the decision-making process occurs.
\end{abstract}

Key words: Cost Drivers; Determinants of Costs; ABC; SCM.

\section{Eric Ferreira dos Santos}

Mestre em Ciências Contábeis pela Universidade Estadual de Maringá (UEM). Graduado em Ciências Contábeis pela Universidade Estadual de Londrina (UEL). Docente do curso de Ciências Contábeis na Universidade Estadual de Londrina (UEL), Universidade do Norte do Paraná (UNOPAR) e Universidade Filadélfia (UNIFIL). Contato: Rodovia Celso Garcia Cid - PR 445 Km 380 Campus Universitário, PR, 86057-970. E-mail: eric_ferreira_net@hotmail.cm

\section{Antonio Ricardo Catânio}

Mestre em Ciências Contábeis pela Universidade Estadual de Maringá (UEM). Especialista em Controladoria e Finanças pela Pontifícia Universidade Católica do Paraná (PUCPR). Graduado em Ciências Contábeis pela Universidade Estadual de Londrina (UEL). Contato: Av. Colombo, 5790, Bloco C-23, Sala 222. Maringá, PR, CEP 87020-900. E-mail: antoniocatanio@gmail.com

\section{Katia Abbas}

Doutora e Mestre em Engenharia de Produção pela Universidade Federal de Santa Catarina (UFSC). Graduada em Ciências Contábeis pela Universidade Estadual de Maringá (UEM). Docente do curso de Ciências Contábeis na Universidade Estadual de Maringá (UEM). Contato: Av. Colombo, 5790, Bloco C-23, Sala 222, Maringá, PR, CEP 87020-900. E-mail:kabbas@uem.br 


\section{INTRODUÇÃO}

Em meados da década de 1980 o mundo corporativo unia seus esforços em busca do aumento da produtividade e desenvolvimento de tecnologia. Com as informações fornecidas pelos métodos de custeios tradicionais, os gestores das empresas tomavam decisões com base em informações distorcidas, insuficientes e alocavam os custos aos produtos de forma arbitrária (Berliner \& Brimson, 1988; Collins \& Werner, 1990; Cooper \& Kaplan, 1988a; Cooper \& Slagmulder, 1998; Miller \& Vollmann, 1985).

Nesta época, Miller e Vollmann, na obra Hidden Factory (1985), apontaram a mão de obra direta e materiais diretos como bases de alocação de overheads aos produtos. Porém Berliner e Brimson (1988), Kaplan e Cooper (1998b) e Shank e Govindarajan (1993) diziam que os métodos tradicionais, que alocavam custos de acordo com bases relacionadas ao volume de produção ou conforme os custos diretos (mão-de-obra direta e materiais), já não eram suficientes para explicarem a distribuição de custos, ou fornecerem informações confiáveis.

Assim, tendo em vista as desvantagens dos métodos tradicionais, tidos como ineficientes, devido à alocação dos custos por meio de cost drivers baseados em volumes, o custeio baseado em atividades (ABC) é projetado para fornecer informações mais precisas e servir de apoio à tomada de decisões dos gestores. Neste sentido, pela complexidade da produção e a necessidade de cost drivers não relacionados ao volume, o ABC amplia os métodos tradicionais, conforme o framework de Kaplan e Cooper (1988a), apresentando os cost drivers não unitários, conhecidos na literatura brasileira como direcionadores de custos (Banker \& Johnston, 2007; Cooper \& Kaplan, 1988a; Kaplan \& Cooper, 1998b).

Os métodos tradicionais no enfoque da Contabilidade de Custos não possibilitavam informações relevantes ao passo que o enfoque da Contabilidade Gerencial carecia de aspectos estratégicos. Assim, o framework proposto por Shank e Govindarajan, denominado Gestão Estratégica de Custos (GEC), contempla a lacuna dos aspectos estratégicos e decisórios dos enfoques mencionados (Shank, 1989; Shank \& Govindarajan, 1993).

Baseado no Strategic Management Framework proposto por Porter e suportado pela teoria econômica industrial, a estrutura conceitual apresentada por Shank e Govindarajan visa o entendimento da estrutura de custos e a busca pela vantagem competitiva pelas empresas. Retirado dos estudos de Porter, um dos pilares que suportam a GEC é a análise de cost drivers (Porter, 1989; Shank, 1989; Shank \& Govindarajan, 1993). Este pilar é denominado na literatura brasileira como determinantes de custos (Costa \& Carneiro, 2014; Slavov \& Takahashi, 2010).

Nesta visão, a compreensão do comportamento dos custos (cost behavior) implica o entendimento da complexa interação do conjunto de cost drivers atuando em uma determinada situação (Shank, 1989; Shank \& Govindarajan, 1993). A princípio, determinantes de custos, no contexto da GEC, são a causa dos custos (Shank \& Govindarajan, 1993), enquanto que, no contexto do $\mathrm{ABC}$, os direcionadores de custos correspondem as bases de alocações de custos aos produtos, serviços e clientes (Cooper \& Kaplan, 1988a; Kaplan \& Cooper, 1998b).

Os cost drivers contemplam terminologias utilizados para diferentes propósitos e a diferença de conceituação dos termos e significativa, segundo abordagem utilizada (Carneiro, 2015). A expressão cost drivers possui variadas terminologias quando traduzida ao português, tais como, determinantes, direcionadores, condutores, geradores de custos, etc., as quais podem confundir o leitor nos contextos e/ou na aplicação de modelos, dado a existência de possíveis vieses (Catânio, 2017).

Por isso, é vital discernir o conceito de cost drivers utilizado em cada estrutura conceitual, verificando a aplicação e a correlação dos termos existentes. Além disso, a clareza da distinção e dos propósitos dos cost drivers pode contribuir para o processo de tomada de decisão pelos gestores das empresas que precisam de informações confiáveis e relevantes. Isto posto, este estudo teve por objetivo realizar uma análise crítica das definições e dos conceitos de cost drivers, considerando os aspectos utilitaristas e de aplicabilidade, bem como os frameworks do ABC e da GEC, conforme expostos na literatura nacional e internacional.

Este assunto requer a ampliação da base teórica, e por isso autores como Banker e Johnston (2007) e Slavov e Takahashi (2010) apontam a necessidade da continuidade do desenvolvimento de teorias subjacentes. Assim, Costa e Carneiro (2014) sugerem a revisão de questões taxonômicas (descrições). No trabalho de Slavov e Takahashi (2010), a proposta foi a de identificar a definição de cost drivers na literatura considerando o contexto da GEC. Desta forma, as discussões teóricas sobre este assunto contribuem para o esclarecimento de conceitos e para a formação de uma base teórica, uma vez que há estudos escassos sobre a temática (Thiel, Rech, Souza, \& Machado, 2017).

Metodologicamente, este estudo caracteriza-se como um ensaio teórico, que consiste na apresentação "lógica e reflexiva e em argumentação rigorosa com alto nível de interpretação e julgamento pessoal” (Severino, 2007, p. 206). Autores de ensaios teóricos buscam a expansão ou o refinamento de constructos, apresentam novas teorias ou analisam as existentes, apontam falhas das teorias ou demonstram vantagens entre elas (American Psychological Association, 2012). Com isso, realizou-se uma pesquisa bibliográfica de materiais nacionais e internacionais sobre o assunto por meio de uma Revisão Narrativa de Literatura (Onwuegbuzie \& Frels, 2016).

\section{ABORDAGEM AMPLA PARA COST DRIVERS}

A inserção de pesquisas na literatura na década de 1980, a partir do desenvolvimento do framework de Cooper e Kaplan (1988a), denominado Activity-Based Costing (Custeio Baseado em Atividades - ABC), e a articulação dos estudos 
de Shank e Govindarajan (1993), referida como Strategic Cost Management (Gestão Estratégica de Custos - GEC), trouxeram concepções das relações das variáveis dos cost drivers em maior nível de detalhe e complexidade, refletindo nos custos das atividades de modo a gerir a alta de overheads e no posicionamento estratégico das empresas em resposta a competitividade.

Cost drivers, a princípio, são apresentados por Blocher, Stout e Cokins (2010) como sendo qualquer fator que possui um efeito de mudança. Aqui, eles expõem a ideia de fatores como mecanismos comportamentais e revelam que o efeito de mudança ocorre no montante do total dos custos. Isto sugere cost drivers como sendo delimitadores de volume dos custos.

Amorim-Melo, Shehab, Kirkwood e Baguley (2014) corroboram com Blocher et al. (2010) atribuindo o adjetivo significante ao termo fator, de forma a evidenciar a existência de uma importância dos cost drivers sobre a mudança nos custos. No entanto, diferentemente de Blocher et al. (2010) que trazem a expressão mudança no montante do total dos custos, Amorim-Melo et al. (2014) focalizam um impacto direto sobre os custos dentro de sistemas.

Apresentados como fatores, os cost drivers podem ser um evento, uma atividade, um processo, um indicador, entre outros, que causam alterações nos custos (Amorim-Melo et al., 2014; Toompuu \& Põlajeva, 2014). Assim, o entendimento dos cost drivers é essencial para o entendimento da estrutura e cost behavior de uma empresa (Cokins, 2002).

Cokins e Căpuşneanu (2010) e Berliner e Brimson (1988) tratam os cost drivers como elementos principais que ditam o cost behavior (comportamento dos custos). Adicionalmente, a complexa interação de um conjunto de cost drivers auxilia na compreensão do cost behavior (Shank, 1989; Shank \& Govindarajan, 1993) que pode ser entendido como efeito mudança.

Cost behavior é entendido como uma relação entre o total de custos e os cost drivers (Sheng, 2009) e uma expressão para descrever se a mudança nos custos altera os outputs (saídas de recursos) (Hansen \& Mowen, 2007). Isto significa que se não houver uma alteração de escolha de outputs não haverá uma mudança no comportamento dos custos.

O efeito mudança está intimamente relacionado com o princípio da causa e efeito. Esta relação ocorre a partir de cost drivers (variáveis) para os custos que são incorridos pelas atividades (Berliner \& Brimson, 1988; Bokor, 2010; Horngren, Datar, \& Rajan, 2014). Por isso, há uma relação de causa e efeito entre fator de custo e montante de custos. Tal relação refere-se aos cost drivers, pois causam mudanças nos custos e impactam nas atividades (Cokins, 2002; El Kelety, 2006; Hansen \& Mowen, 2007; Horngren et al., 2014), os quais guardam uma relação de homogeneidade de custos (Chea, 2011). As oscilações que ocorrem nas atividades são observadas através de eventos (Cokins, 2002), e, com base nos parágrafos anteriores, é possível inferir que estas oscilações são observadas a partir das variações nos montantes de custos.

A Figura 1 exibe a definição genérica de cost driver a partir dos conceitos expostos.

Figura 1- Representação da definição genérica de cost driver

$\begin{array}{lll}\text { Cost driver } & \text { Cost behavior/relação causa-e-efeito/efeito mudança } & \text { Volume/montante de custos } \\ \end{array}$

Fonte: elaborada pelos autores

Cokins (2002), El Kelety (2006), Hansen e Mowen (2007), Sheng (2009) e Horngren et al. (2014) explicam o comportamento dos custos através dos cost drivers, o qual relacionam estes com os montantes de custos e com as atividades. Além de apontarem os cost drivers como o principal determinante significativo de custos, Berliner e Brimson (1988) adicionam que as decisões devem ser consideradas e que os cost behaviors, para serem explicados, dependem dos (1) cost drivers, (2) das atividades e (3) das decisões.

Para Geiger (1999), existem diversas possibilidades de decisões para a escolha de cost drivers. Estas escolhas podem acarretar distribuições de custos de maneira diferente, representar novos modos de mensuração, e fazer distintas suposições ligadas a reações comportamentais. Ainda de acordo com o autor, a escolha de um cost driver deve ser credível e considerar o custo/benefício de obtê-lo e atualizá-lo (Geiger, 1999).

Estas diferentes possibilidades de escolha são apresentadas na Figura 2, que a princípio foi apresentada por Lyly-Yrjanâinen, Kulmala e Paranko (2000) visando exibir o modo de como os custos são atribuídos, considerando a abordagem do ABC. No entanto eles, ao descreverem o processo, iniciaram mencionando a existência do cost factor (determinantes de custos/cost drivers) e, em seguida, apresentaram as atribuições de custos por meio de resource drivers e activity drivers (direcionadores de custos/cost drivers)

As possibilidades de escolhas de cost drivers consideram os seguintes atributos: relações de causa-e-efeito ou entre váriaveis; comportamento dos custos ou mecanismos comportamentais; e volume de custos ou montante de custos. Enquanto a proposta de Lyly-Yrjanâinen et al. (2000) mostrou as atribuições dos custos, a proposta dos autores do presente estudo é apresentar as possíveis relações de cost drivers por meio das váriaveis A, B, C e D. 
Figura 2 - Possibilidades de comportamentos dos cost drivers

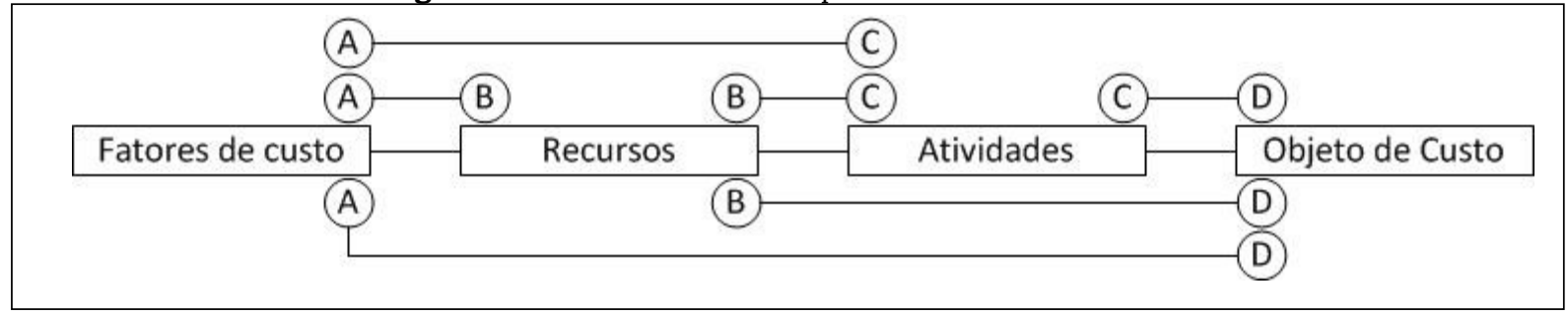

Fonte: adaptado de Lyly-Yrjanâinen et al. (2000)

As relações entre as variáveis $A, B, C$ e D remetem ao conceito exposto na Figura 1. As relações $A B, A C, A D, B C$, $\mathrm{BD}$ e $\mathrm{CD}$ representam as possibilidades de escolhas de cost drivers. Lyly-Yrjanâinen et al. (2000) trazem fatores de custos como atribuições aos recursos $(\mathrm{AB})$, os recursos como atributos às atividades $(\mathrm{BC})$ e as atividades como atributos aos objetos de custos (CD). Hansen e Mowen (2007) aludem que os custos reagem com as mudanças das saídas de recursos de diversas maneiras. A princípio isto se refere à $\mathrm{BD}$, como visto em Chea (2011), em que os recursos refletem uma relação de causa e efeito com os objetos de custos (BD). Contudo, é possível a associação de $\mathrm{C}$ à $\mathrm{D}$ conquanto que traga a ideia de recursos das atividades em $\mathrm{C}$, pois o termo recursos está no sentido amplo podendo se remeter a $\mathrm{B}$.

De outro modo, a relação CD inicia-se das atividades aos objetos de custos por meio de activity drivers que mensuram saídas (Cokins, 2002) e C pode ser expressa de diversas maneiras: custos das atividades, recursos das atividades, atividades, alteração no nível de atividades, mudanças nas atividades, entre outras expressões similares que retornem a mesma concepção. Sendo assim, El Kelety (2006) e Blocher et al. (2010) defendem que há uma relação de causa e efeito entre a atividade e os custos totais de um objeto de custo (CD).

Porter (1985, p. 63), ao apontar que cost drivers são "the structural determinants of the cost of an activity", realizou uma relação $\mathrm{AB}$ e $\mathrm{AC}$, pois determinantes estruturais referem-se a fatores de custos (A) ou de uma ordem superior (higher order) em relação à causa principal (Cokins, 2002). Conforme o autor, custo refere-se a insumos que são recursos (B), e o custo de uma atividade consiste em um fluxo de recursos para as atividades (C).

Cokins (2002) comenta acerca de cost driver como um agente responsável pela causa de uma atividade existir (AC) e consumir recursos para a realização de processos (BC). “What causes cost” (Cokins, 2002, p. 49; Shank, 1989, p. 55) é a expressão usada pelos autores para questionar a causa dos custos (determinantes de custos) de uma atividade. $\mathrm{O}$ consumo de recursos é tratado por Cooper e Kaplan (1992), dentro do contexto do ABC, como sendo recursos utilizados para a realização das atividades. Estas utilizações de recursos são direcionadas para as atividades (Cokins, 2002).

Blocher et al. (2010), ao utilizar a terminologia montante de custos, deixa subjetivo o emprego do termo. Como visto, custos podem representar os insumos, os recursos ou até mesmo os custos das atividades. Sugere-se a especificação para custos dos recursos (B) ou para custos das atividades (C). Por fim, em uma relação AD, os determinantes de custos configuram-se em observância aos objetos de custos, ambos compreendidos a partir do conjunto das possibilidades de escolhas de cost drivers apresentadas até o instante.

As relações utilizadas para explicar os cost drivers, neste estudo, consideram os frameworks do ABC e da GEC. Na literatura brasileira, o termo cost drivers, ao ser aplicado nestes contextos, recebe vieses tanto na tradução quanto no emprego do conceito.

\section{VIESES DO TERMO COST DRIVERS}

A literatura brasileira reconhece diversos sinônimos de traduções para o termo cost drivers aplicado para diversos conceitos (Costa, 2011; Costa \& Carneiro, 2014; Panarella, 2010; Santos, 2014; Slavov, 2013; Slavov \& Takahashi, 2010; M. A. De Souza \& Mezzomo, 2012). Cokins (2002) coloca que o termo driver tem diferentes variações e aplicações com significados distintos. Pelas publicações nacionais, entende-se que o termo cost drivers possui os sentidos de: determinantes de custos e direcionadores de custos.

Porter (1985), ao usar o conceito de cost drivers para explicar o comportamento dos custos, se remeteu aos conceitos de determinantes de custos. A obra original apresenta: "Cost drivers are the structural determinants of the cost of an activity" (Porter, 1985, p. 63). Porém, a obra traduzida do referido autor traz cost drivers como "condutores dos custos" (Porter, 1989, p. 58).

Shank (1989) e Shank e Govindarajan (1993), baseados nos estudos de Porter (1985), ao abordarem a literatura sobre a GEC apontam três temas sendo o terceiro denominado “Cost driver analysis" (Shank, 1989, p. 50; Shank \& Govindarajan, 1993, p. 13). Todavia, a obra traduzida fornece, respectivamente, as seguintes traduções: "Análise de direcionadores de custos" (Shank \& Govindarajan, 1997, p. 13) e "O Conceito de Direcionador de Custos" (Shank \& Govindarajan, 1997, p. 21). Apesar de traduzido como direcionador, neste contexto o termo apresenta-se com o conceito de determinantes.

Kaplan e Cooper (1998a, p. 92), ao utilizarem cost drivers nos estudos de ABC, aludem que os custos "must be driven to products, services or customers by a single cost driver", ao passo que no livro traduzido, os custos "devem ser alocados a produtos, serviços ou clientes por um único gerador de custos” (Kaplan \& Cooper, 1998b, p. 106). Observa-se na tra- 
dução a expressão gerador de custos, reportando-se aos direcionadores de custos. De modo similar, o termo gerador de custos é utilizado em Berliner e Brimson (1992) na obra traduzida, enquanto que no texto original encontra-se cost drivers (Berliner \& Brimson, 1988).

Hansen, Mowen e Guan (2007) apresentam duas categorias para os cost drivers: os organizacionais e os operacionais. Compreendidos como determinantes de custos, cost drivers organizacionais são apresentados no livro, e traduzidos, como direcionadores de custos (Hansen \& Mowen, 2003). E os operacionais são apresentados e entendidos como direcionadores de custos na mesma obra. De outra maneira, Nakagawa (1994, p. 74), ao apresentar cost drivers como rastreadores de custos dentro do framework do $\mathrm{ABC}$, apresenta um sinônimo do termo: vetores de custos.

Em síntese, os sinônimos das possíveis traduções de cost drivers presentes na literatura brasileira são: condutores, determinantes, geradores, direcionadores, vetores e rastreadores. Autores como Costa e Carneiro (2014) listaram estes termos sem relacioná-los aos autores da literatura, em contraste com Mário (2013), Souza e Mezzomo (2012) e Wrubel (2009) que relacionaram os termos aos autores da literatura.

Rocha (1999), ao analisar os estudos de Shank e Govindarajan (1997) e observar o termo direcionadores, coloca que determinante é preferível a direcionador sem justificar a preferência pelo termo, embora esclareça o seu conceito. Ao contrário disso, Mário (2013) justifica o uso do termo para referir-se ao conceito de fatores estruturais e execucionais. Em relação a estes estudos de Shank e Govindarajan (1997), Rocha (1999) menciona que os autores utilizam a expressão determinantes de custos, porém tal expressão não é encontrada nos trabalhos destes autores, conforme já informado anteriormente.

Diehl, Miotto e Souza (2012) encontraram as traduções de cost drivers em Porter (1989) e Shank e Govindarajan (1997), compreenderam direcionadores de custos como sinônimo do termo e conceito de determinantes, tal como Slavov (2013) e Wrubel (2009), e optaram por determinantes de custos, com base em Rocha (1999). Além de se confundirem com os termos, mas não com o conceito, corroboraram com a opção dada por Rocha (1999) para o termo.

Slavov e Takashi (2010) propuseram a discussão sobre as definições de cost drivers encontradas na literatura internacional e brasileira. Em relação à literatura internacional, não apresentaram as definições e os conceitos relevantes propostos por Porter (1985), Shank e Govindarajan (1993), Kaplan e Cooper (1998a), entre outros. Sob a ótica da literatura brasileira, Slavov e Takashi (2010) entenderam determinantes e direcionadores como traduções de cost drivers, ao contrário de Costa e Carneiro (2014) que apresentaram estes termos como sinônimos entre si. Ressalta-se o uso adequado entre tradução, sinônimo e definição, pois determinantes e direcionadores referem-se a possíveis traduções de cost drivers com definições distintas acerca dos cost drivers, logo não são sinônimos.

Costa e Carneiro (2014) corroboram, incluindo aspectos da vantagem competitiva, com a definição de determinantes estabelecida por Slavov e Takashi (2010) que é exposta como precisa para explicar o conceito de determinantes de custos. Percebe-se que há uma ausência de fundamentações teóricas para suportar e indicar o uso das palavras utilizadas na composição da definição. Também, verifica-se uma fragilidade na definição de direcionadores ao apontarem a finalidade de obtenção de uma evolução da discussão conceitual (Slavov \& Takahashi, 2010) sem evidenciar a evolução dos conceitos em uma série.

Reconhecendo as possibilidades de emprego de cost drivers nos frameworks do ABC e da GEC, Costa (2011), Mário (2013) e Panarella (2010) sugeriram determinantes de custos como: maior fator constituinte na causa real do custo; melhor expressão para imputar por fatores causais; e variáveis explicativas do cost behavior, respectivamente. E recomendaram direcionadores de custos como: a mensuração do custeamento de uma atividade; fatores que conduzem custos às atividades; e medidas intencionais adotadas para atingir a eficácia e a eficiência, respectivamente. Os conceitos destes termos são tratados a seguir.

\section{COST DRIVER - DETERMINANTES DE CUSTOS}

\subsection{Conceito de Determinante de Custos}

Os autores deste artigo compreendem que Porter $(1985,1989)$, ao referir-se a cost drivers/condutores dos custos, tratou o conceito de determinantes de custos. Segundo ele, condutores de custos são "determinantes estruturais do custo de uma atividade, e diferem de acordo com o controle que uma empresa exerce sobre eles. Eles determinam o comportamento dos custos dentro de uma atividade, refletindo quaisquer elos ou inter-relações que o afetem” (Porter, 1989, p. 58).

Apresentado o conceito de cost drivers, Porter (1985, p. 70, 1989, p. 62) denomina as "causas estruturais dos custos" como sinônimo de "determinantes estruturais dos custos". Para ele, os determinantes se diferenciam conforme o controle exercido pela empresa e distingue-se entre empresas do mesmo setor. Eles podem estar (ou não) sob controle de uma entidade, interagem para determinar o cost behavior e o custo de uma atividade e recebem atribuições de importâncias. Os determinantes influenciam o desempenho dos custos das atividades e o conjunto de desempenhos compõe a posição dos custos de uma empresa, a qual é resultante do cost behavior. Há ainda uma relação de dependência entre a posição dos custos e cost drivers significativos (Dixon \& Smith, 1993; Porter, 1985, 1989).

Shank e Govindarajan (1993, p. 19, 1997, p. 21) reconhecem que "o custo é causado, ou direcionado, por muitos fatores que se inter-relacionam de formas complexas. Compreender o comportamento dos custos significa compreender a complexa interação do conjunto de direcionadores de custo em ação em uma determinada situação”. A menção 
de que o custo é causado, remete às causas estruturais de Porter. E ao tratarem dos fatores que se inter-relacionam de forma complexa aludem a interação entre os determinantes de custos, assim como visto em Porter. Todos estes autores retratam a associação entre o cost behavior e os cost drivers. Complementando, Banker e Johnston (2007) argumentam que o cost behavior é entendido em função das escolhas estratégicas (variáveis de decisão) e deve envolver a análise de toda cadeia de valor (Banker \& Johnston, 2007; Porter, 1985; Shank, 1989; Shank \& Govindarajan, 1993).

Cokins e Căpuşneanu (2010), Dixon e Smith (1993), e El Kelety (2006) contribuem com a construção do conceito de determinantes de custos pelo entendimento de que cost drivers (determinantes) são fatores causais que determinam ou produzem os custos das atividades. Lord (1996) declara que as causas dos custos são chamadas de cost drivers.

Para Cokins (2002), cost driver (determinantes) é um driver de uma ordem superior aos activity drivers (direcionadores). Esclarece cost driver (determinantes) como não sendo necessariamente mensurável, mas podendo de modo simples ser descrito como um triggering event (ponto de partida de um evento). Enquanto Porter (1985) e Shank e Govindarajan (1993) tratam a interação dos cost drivers influenciando uma atividade, Cokins (2002) discorre sobre a capacidade de um único cost driver afetar múltiplas atividades.

A gestão dos determinantes de custos pode não ser tão crítica as vezes, contudo a atenção ao conjunto de determinantes contribui para o sucesso da empresa. Por exemplo, o varejo tem a perda e os danos à mercadoria, que reduzem o desempenho econômico e a eficiência, como um relevante determinante de custos (Blocher et al. 2010). Considerando isto, muitos varejistas podem estabelecer processos para a manipulação e a armazenagem suas mercadorias.

Souza e Rocha (2009, p. 37) definem determinantes de custos como "fatores que determinam a existência ou ausência de um elemento de custo, definem seu limite inferior e seu comportamento e influenciam a composição da estrutura de custos de uma entidade”. Estes autores evidenciam a ocasião da existência ou ausência (dummy variable) de um elemento de custo sendo explicado por um fator (determinante). Corroboram com Porter (1985), Shank (1989) e Shank e Govindarajan (1993) no apontamento das influências na estrutura dos custos de uma empresa apresentando a expressão composição, geralmente entendida como o conjunto de custos fixos e variáveis (Blocher et al., 2010; Cokins, 2002; Cooper \& Kaplan, 1991, 1992; Miller \& Vollmann, 1985).

Costa e Carneiro (2014, p. 4) entendem que determinantes são "as causas reais dos custos. [...], a raiz dos custos, [...], os elementos que estão no cerne e provocam a existência de um determinado custo, sua essência”. Em análise, pode-se inferir parte desta definição como sendo fundamentada de Lord (1996) e Souza e Rocha (2009). A raiz dos custos possui uma ideia semelhante com a de autores, como Cokins (2002) e Lebas (1999), ao colocarem que os custos possuem uma causa raiz (root cause). Ainda, vê-se a preocupação de Costa e Carneiro (2014) em associar os determinantes de custos com a origem dos custos.

\subsection{Utilidade dos Determinantes de Custos}

Sob o aspecto utilitarista, não se gerenciam os custos, mas as causas dos custos (cost drivers/determinantes), a eficácia e a eficiência da organização. Por exemplo, o evento de promoção de vendas pode ser um cost driver (determinante) por envolver um aumento das atividades de uma empresa (Cokins, 2002). Deste modo, tal evento pode ser gerenciado observando a estrutura instalada de forma a gerar ou reduzir custos (causa e efeito).

Com base nos conceitos, compreende-se que os determinantes, quando analisados, são úteis para: o entendimento da posição relativa dos custos; a identificação das interações entre os cost drivers e o comportamento dos custos; e a compreensão da estrutura de custos.

\subsection{Aplicações dos Determinantes de Custos}

No contexto da GEC, para a análise dos cost drivers, inicialmente identifica-se a cadeia de valor segregando-a em atividades de valor da empresa e atribui-se ativos e custos operacionais a elas. Em seguida, deve-se diagnosticar os cost drivers (Hansen \& Mowen, 2007; Porter, 1985; Shank, 1989; Shank \& Govindarajan, 1993). O Open Book Accounting (OBA), por exemplo, pode ser utilizado para identificar cost drivers na cadeia de valor (Hoffjan \& Kruse, 2006).

O diagnóstico, segundo Porter (1985), consiste em identificar os cost drivers e suas interações, que podem ser reforçadoras e neutralizadoras. A identificação pode ser vista claramente de forma intuitiva por meio de: i) exame da experiência interna da empresa; ii) entrevistas com especialistas; e iii) comparação dos custos dos concorrentes. A identificação dos cost drivers e a quantificação do seu impacto sobre os custos podem não ser simples e diversos métodos podem ser utilizados, tal como a análise de regressão, útil para quantificar o impacto financeiro dos drivers (Porter, 1985; Shank \& Govindarajan, 1993). Bjørnenak (2000) reforça e complementa ao listar que os determinantes de custos podem ser identificados por meio de: i) análise de regressão; ii) análise da atividade; iii) análise do cost driver de estrutura; e iv) análise dos atributos dos produtos. Bjørnenak (2000) comenta que o uso de entrevistas e discussão como administrações contribui para esse processo.

Identificados os cost drivers, estes podem ser categorizados em listas, tais como as de Porter (1985) e Riley (1987) apud Shank e Govindarajan (1993), entre outros. Porém há uma ausência de uniformidade quanto à relação e à classificação dos determinantes de custos, pois a variedade de fatores pode gerar listas extensas e quando comparadas entre si é possível verificar discrepâncias de fatores revelando a falta de consenso em relação ao tema. Com isto, a literatura 
reconhece a necessidade de resumir e organizar as diversas listas existentes (Banker \& Johnston, 2007; Costa \& Carneiro, 2014; Costa \& Rocha, 2014).

Na literatura da GEC, Porter (1985), Riley (1987) apud Shank e Govindarajan (1993), Hansen et al. (2007) e Souza e Rocha (2009) apresentaram listas de cost drivers (determinantes) e, dentre os itens listados estão: escala, escopo, tecnologia, elos, inter-relações, localização, modelo de gestão, gestão da qualidade, layout, estrutura de capitais. Esta categorização foi adotada para o contexto da GEC por Shank e Govindarajan (1993).

Análises desses fatores nas empresas são fundamentais para compreender o impacto que as escolhas estratégicas terão nos custos. Por exemplo, no setor de aviação, a tecnologia é tida como um relevante determinante de custos porque pode reduzir o custo com pessoal, aumentar a escala de produção, melhorar a qualidade dos serviços, ou mesmo, se a tecnologia fora inadequada ou excessiva, pode gerar custos adicionais (Diehl et al. 2012). Um determinante de custos pode se sobressair em relação aos demais determinantes porque eles não são igualmente importantes (Shank \& Govindarajan, 1993). Assim, uma empresa de aviação que tem alta dependência da tecnologia terá esse fator como um relevante determinante de custos.

A análise de cost drivers na GEC possui uma abordagem mais ampla que a análise vista no contexto do $A B C$, tido como uma ferramenta útil de análise estratégica que focaliza primeiramente em complexidade (Kaplan \& Cooper, 1998a; Lord, 1996; Shank, 1989; Shank \& Govindarajan, 1993). Ao passo que na GEC os cost drivers são tratados como determinantes, no $A B C$ vê-se pelo conceito de direcionadores.

\section{COST DRIVER - DIRECIONADORES DE CUSTOS}

\subsection{Conceito de Direcionador de Custos}

Por volta de 1980, as empresas distribuíam os overheads aos produtos utilizando bases de alocação tradicionais (geralmente alocações baseadas em volume e mão de obra direta) e não se discutiam a necessidade de outras bases. Com os avanços do método $\mathrm{ABC}$, tendo em vista o aumento e a complexidade do sistema produtivo, e, consequentemente, o aumento dos overheads, surgia a necessidade de discussões das bases de alocações. A partir da publicação Hidden Factory, de Miller e Vollmann (1985), o termo base de alocação sofreu desuso em virtude do termo cost driver (Cokins \& Căpuşneanu, 2010; Collins \& Werner, 1990; Cooper \& Kaplan, 1992; Kaplan \& Cooper, 1998a; Miller \& Vollmann, 1985).

$\mathrm{O} A B C$ é uma ferramenta de gestão que classifica e separa os custos dos recursos, das atividades e dos objetos de custos com base em informações financeiras e não-financeiras. A atribuição e o rastreamento de recursos para as atividades baseadas em consumo, e também, dos custos das atividades aos objetos de custo (produto, serviços ou clientes) compõem as ações dos cost drivers. Primeiro ocorre o relacionamento entre os recursos e as atividades, depois entre as atividades e os objetos de custos (Chea, 2011; Cokins \& Căpuşneanu, 2010; Kaplan \& Cooper, 1998a; Perčević \& Lutilsky, 2008).

As atribuições, compreendidas conceitualmente pelas relações, são vistas como alocações ou distribuições de recursos tidas, no Brasil, pelo termo direcionadores de custos. Os direcionadores causam mudanças nos custos dos elementos de destino (atividades e objetos de custo) por meio do consumo de recursos (Babad \& Balachandran, 1993; Cokins \& Căpuşneanu, 2010; Costa \& Carneiro, 2014; Estermann \& Claeys-Kulik, 2013; Kaplan \& Cooper, 1998a; Nakagawa, 1994).

Os cost drivers, conhecidos por bases de alocação no método do ABC, são medidas de atividades, consumidoras de recursos de pouca ou grande quantidade, que possuem um efeito mensurável e podem ser custeados. Empresas empregam o critério de causa e efeito para alocar custos. As alocações de custos partem de uma única fonte para diversas unidades de distribuições, então são redistribuídas proporcionalmente das atividades para os objetos de custos através de drivers (Blocher et al., 2010; Cokins \& Căpuşneanu, 2010; Cokins, 2002; Estermann \& Claeys-Kulik, 2013; Geiger, 1999; Horngren et al., 2014; Lyly-Yrjanâinen et al., 2000; Noreen, 1991).

No ABC, conforme Alcouffe, Berland, Dreveton e Essid. (2010), o termo cost drivers é tido como confuso dado à existência de tipos de direcionadores. Cokins (2002) apresenta três tipos de direcionadores que requerem serem quantitativos: resource drivers, activity drivers e cost object drivers. Os direcionadores de recursos rastreiam custos dos recursos às atividades; os drivers de atividades direcionam os custos das atividades aos objetos de custos; e os object drivers conduzem custos dos objetos de custos a outros objetos. Os resource drivers e activity drivers estão representados na Figura 2 respectivamente pelas relações: $\mathrm{BC}, \mathrm{CD}$.

\subsection{Utilidade dos Direcionadores de Custos}

Em resposta às limitações trazidas pelas abordagens de custeios tradicionais e a necessidade de explicar ou predizer os comportamentos dos custos, o ABC fornece uma informação mais acurada e útil ao processo decisório por meio da aplicação de múltiplos direcionadores de custos para as alocações de custos (Schniederjans \& Garvin, 1997).

Os direcionadores de custos são úteis para mensurar a utilização de overheads e prover informações com maior grau de acurácia quando utilizado um elevado número de cost drivers (Homburg, 2001). Cokins e Căpuşneanu (2010) listam utilidades da aplicação dos drivers: melhoria do desempenho da empresa, conscientização dos funcionários e gerentes, revisão periódica dos custos, controle dos custos com melhores cálculos, eliminação de custos e determinação do custo 
efetivo do produto. A revisão dos custos e o entendimento da relação entre cost drivers e cost behavior auxilia na compreensão do montante de custos (Cokins \& Căpuşneanu, 2010; Cokins, 2002).

\subsection{Aplicações dos Direcionadores de Custos}

A seleção de cost drivers implica na qualidade das informações, pois Toompuu e Põlajeva (2014) e Babad e Balachandran (1993) entendem a necessidade do uso de múltiplos drivers visando resultados mais precisos, tal como Cokins (2002). Contudo a escolha dos drivers, baseada no princípio da decisão relevante, requer cautela e consciência sem que haja a necessidade da relação acurácia/correção/mensuração dos custos (Pingxin, Fei, Dinghua, \& Lin, 2010; Toompuu \& Põlajeva, 2014). Ao tratar da seleção dos activity drivers, Cokins (2002) fornece recomendações: a abstenção do uso de drivers para valores imateriais, seleção de drivers com alta correlação, restrição da quantidade de drivers, escolhas promotoras de melhorias no desempenho e seleção de drivers de fácil medição. A seleção de drivers pode ser modelada utilizando os métodos quantitativos Analytic Hierarchy Process (AHP), Zero-One Goal Programming (ZOGP), regressão e correlação (Bokor, 2010; Schniederjans \& Garvin, 1997). São exemplos de direcionadores que podem ser escolhidos: horas-máquina; horas de manutenção; e horas de preparação (Kaplan \& Cooper, 1998a).

Neste contexto, a análise dos cost drivers consiste no exame, quantificação e explicação das relações de causa e efeito entre os drivers e o total de overheads de uma operação. O fato de o cost driver envolver o cost behavior e este influenciar no montante dos overheads, propicia a viabilidade e a precisão das informações no sentido de apoiar as empresas nas decisões estratégicas (Bokor, 2010; Cokins \& Căpuşneanu, 2010; Schniederjans \& Garvin, 1997). Os cost drivers, depois de selecionados, geralmente por meio de métodos matemáticos e estatísticos, servirão para alocar os custos às atividades/objetos de custos.

\section{COST DRIVER - DETERMINANTES E DIRECIONADORES}

Com base no exposto até esta seção, o Quadro 1 sintetiza o entendimento do termo e do conceito entre determinantes e direcionadores de custos.

Quadro 1 - Características dos Cost drivers conforme frameworks GEC e ABC

\begin{tabular}{|c|c|c|}
\hline & Determinante de custos & Direcionador de custos \\
\hline Framework & Gestão Estratégica de Custos (GEC) & Custeio Baseado em Atividades (ABC) \\
\hline Palavras-chave conceituais & $\begin{array}{l}\text { Origem; causas estruturais; triggering } \\
\text { event, dummy event, raizes dos custos }\end{array}$ & $\begin{array}{l}\text { Bases de alocação; medidas de atividades; } \\
\text { distribuidores }\end{array}$ \\
\hline Possíveis sinônimos & Gerador / Causador / Fato gerador & Condutor / Vetor / Alocador / Rastreador \\
\hline Cost behavior & Delimita o montante total dos custos & $\begin{array}{l}\text { Distribui o custo para a atividade e redistribui ao } \\
\text { objeto de custo }\end{array}$ \\
\hline Utilidade & $\begin{array}{l}\text { Gerenciar a eficácia e a eficiência e não } \\
\text { os custos }\end{array}$ & $\begin{array}{l}\text { Mensurar e prover informações } \\
\text { com maior grau de acurácia }\end{array}$ \\
\hline Método de análise & $\begin{array}{l}\text { Análise de regressão, análise da atividade; } \\
\text { análise do cost driver de estrutura, análise } \\
\text { dos atributos dos produtos }\end{array}$ & Regressão, correlação, AHP, ZOGP \\
\hline Relações na Figura 2 & $\mathrm{AB} / \mathrm{AC} / \mathrm{AD}$ & $\mathrm{BC} / \mathrm{CD} / \mathrm{BD}$ \\
\hline Natureza & Quali-quantitativo & Quantitativo \\
\hline
\end{tabular}

Fonte: elaborado pelos autores

Conforme o Quadro 1, existem características específicas dos cost drivers classificados nos frameworks da GEC, tendo como autores recorrentes nestas literaturas Porter (1985) e Shank e Govindarajan (1993), e do ABC, Cooper e Kaplan (1998a) e Cokins (2002). Em se tratando dos determinantes têm-se como norteadoras as palavras-chave origem e causas, pois estas indicam as raízes ou fatos geradores dos custos. Estrategicamente, os determinantes delimitam o montante do total dos custos no sentido de gerenciar a sua eficácia e eficiência e são compreendidos nas relações $\mathrm{AB} / \mathrm{AC} / \mathrm{AD}$. Quanto às abordagens dos determinantes, têm-se a possibilidade de se apresentarem como qualitativas, quantitativas ou quali-quantitativas.

Os direcionadores de custos distribuem os custos às atividades e redistribuem aos objetos de custos. Eles mensuram e provêm informações com maior grau de acurácia aos gestores e apresentam-se de diversas formas no ABC, contudo, no Time-driven activity-based costing (TDABC), encontram-se sob uma única forma: o time-driver (Kaplan, 2014). Assim, os direcionadores são tidos como alocadores representados nas relações $\mathrm{BC} / \mathrm{CD} / \mathrm{BD}$. Em relação à natureza, o mesmo não ocorre para os direcionadores, que são apenas quantitativos. 
Cokins (2002) argumenta que um cost driver não necessariamente é descrito em números, mas pode ser expresso por palavras. O autor traz o exemplo de que um tornado pode ser um cost driver caso provoque bastante trabalho de limpeza e, consequentemente, origine custos. Esta ideia remete-se ao conceito de determinantes. Por outro lado, segundo o autor, drivers que recebem atribuições de custos do $\mathrm{ABC}$ devem necessariamente ser quantitativos por utilizarem medidas de (re)distribuição de custos. Toompuu e Põlajeva (2014) revelam a possibilidade de cost driver atuando como determinante e direcionador.

Com base neste estudo, visando enriquecer a base teórica, propõem-se as seguintes definições: i) determinante de custos é a escolha estratégica ou o fato gerador de uma ordem superior de cost drivers que indica a condição de existência ou ausência do custo, pode ser expresso por informação qualitativa e/ou quantitativa e influência no comportamento e no montante de custos; ii) direcionador de custos é a base de alocação que distribui recursos às atividades e redistribui custos aos objetos de custos, sendo uma informação quantitativa e serve como medidas de desempenho, de caráter operacional. Pelos aspectos dos cost drivers retratados, os determinantes de custos podem definir os rumos estratégicos enquanto os direcionadores fornecem informações mais acuradas, confiáveis e operacionais.

As relações utilizadas para explicar os cost drivers, neste estudo, consideram os frameworks do ABC e da GEC, no entanto Alcouffe et al. (2010), Banker e Johnston (2007) e Bjørnenak (2000) apontam a existência de outras abordagens para explicá-los. Assim, Banker e Johnston (1993), Foster e Gupta (1990), Datar, Kekre, Mukhopadhyay e Srinivasan (1993), Homburg (2001) fornecem diferentes visões sobre os drivers.

\section{CONSIDERAÇÕES FINAIS}

Este trabalho objetivou realizar uma análise crítica das definições e dos conceitos de cost drivers, considerando os aspectos utilitaristas e de aplicabilidade, bem como os frameworks do ABC e da GEC, conforme expostos na literatura nacional e internacional. Para tanto, discorreu-se acerca dos cost drivers em sentido amplo, abordou-se os vieses das terminologias de determinantes e direcionadores de custos, e conceituou-os trazendo à tona os aspectos utilitaristas e de aplicabilidade. Tal discussão foi essencial para o aprofundamento do tema e o aperfeiçoamento dos conceitos, sem a intenção de esgotar todo o assunto, considerada a importância dos cost drivers nos frameworks do ABC e da GEC.

Em síntese, considerando as análises efetuadas, cost driver, em termos gerais, é corresponde a um fator ou evento, o qual possui um efeito mudança que altera o montante dos custos. O efeito mudança representa o comportamento dos custos que por sua vez é a relação dos cost drivers com o total dos custos. Determinantes de custos são cost drivers de uma ordem superior de drivers e representam uma variável condicional de existência do custo, em outras palavras, são fatores geradores de custos ou recursos atuando como gerenciadores da eficácia e da eficiência dos custos. Por sua vez, direcionadores de custos são concebidos como bases alocadoras que distribuem custos às atividades e redistribuem aos objetos de custos, servindo de medidas de desempenho.

Quanto aos aspectos utilitários, tem-se que os determinantes de custos devem ser considerados no processo decisório já que estão ligados à gestão da eficácia e a eficiência, e não aos custos em si, sendo estratégicos. Nesse sentido, um determinante de custos contribui na definição de rumos estratégicos pelas escolhas estratégicas. Uma empresa pode decidir se instalar na cidade A ou B, por exemplo. A escolha pela localização implicará em um montante de custos em níveis maiores ou menores, dado às razões tributárias, logísticas e mercadológicas. Por esses aspectos, podem ser difíceis de mensurar, embora haja diversos caminhos de análises. Os determinantes de custos indicam as escolhas estratégicas da empresa, seja pela característica de um setor econômico ou por uma necessidade de atuação no mercado.

Por outro lado, os direcionadores de custos visam mensurar e prover informações com maior grau de acurácia, impactando na distribuição ou alocação dos montantes de recursos previamente definidos como consequência das escolhas estratégicas. Neste ponto, os direcionadores complementam a função dos determinantes. Os direcionadores atribuem relações acuradas entre montantes de recursos, visando a destinação de uma quantidade específica de custos para uma atividade de valor ou para um objeto de custos.

Essas observações tornam-se relevantes por ser crescente a necessidade de análises estratégicas voltadas para uma gestão estratégica de custos e operacional de modo a contribuir para o processo decisório.

Destaca-se que a literatura dos direcionadores de custos é mais difundida que a dos determinantes. Isto se deve à popularização do framework do ABC de Kaplan e Cooper a partir da década de 1980 (Cooper \& Kaplan, 1988a, 1988b, 1991; Kaplan \& Cooper, 1998a). Esses autores são tidos por Cokins (2002) como propagadores dos conceitos do ABC e não como criadores. Já, a literatura dos determinantes teve repercussão após a publicação da obra Strategic cost management de Shank e Govindarajan (1993) na década de 1990, época em que a Contabilidade Gerencial ganhou enfâse estratégica.

A terminologia utilizada para determinantes possui vieses, que podem levar à confusão dos termos voltados para o direcionador, por isso é necessário a compreensão dos conceitos dos dois tipos de cost drivers. Assim, após as críticas, sintetizou-se as características, propondo definições a fim de contribuir para a formação da base conceitual sobre cost drivers. Pesquisadores devem tomar sempre o cuidado no uso dos conceitos e termos para os cost drivers, de modo a evitar conflitos conceituais.

Sugere-se para futuras pesquisas, a realização de estudos em campo sobre cost drivers e, em especial, sobre determinantes de custos, pois esta temática ainda carece de evidências empíricas e de direcionamentos práticos quanto à sua mensuração. Ainda, pesquisas de cunho teórico que abordem os aspectos de outros frameworks não retratados nesta pesquisa podem ser abordadas em estudos futuros. 


\section{REFERÊNCIAS}

Alcouffe, S., Berland, N., Dreveton, B., \& Essid, M. (2010). An Empirical Study Of Environmental Cost Drivers. Crises et Nouvelles Problématiques de La Valeur.

American Psychological Association. (2012). Manual de publicação da APA (6th ed.). Porto Alegre: Penso.

Amorim-Melo, P., Shehab, E., Kirkwood, L., \& Baguley, P. (2014). Cost Drivers of Integrated Maintenance in High-value Systems. Procedia CIRP, 22, 152-156.

Babad, Y. M., \& Balachandran, B. V. (1993). Cost driver optimization in activity-based costing. The Accounting Review, 68(3), 563-575.

Banker, R. D., \& Johnston, H. H. (1993). An empirical study of cost drivers in the US airline industry. Accounting Review, 68(3), 576-601.

Banker, R. D., \& Johnston, H. H. (2007). Cost and profit driver research. In Handbook of Management Accounting Research (Vol. 2, pp. 531-556). Oxford: Elsevier.

Berliner, C., \& Brimson, J. A. (1988). Cost management for today's advanced manufacturing: the CAM-I conceptural desing. Boston: Harvard Business School Press.

Berliner, C., \& Brimson, J. A. (1992). Gerenciamento de custos em indústrias avançadas: base conceitual CAM-I. (J. L. Basetto, Trans.). São Paulo: T.A. Queiroz.

Bjørnenak, T. (2000). Understanding cost differences in the public sector-a cost drivers approach. Management Accounting Research, 11(2), 193-211.

Blocher, E. J., Stout, D. E., \& Cokins, G. (2010). Cost Management: A Strategic Emphasis (5. ed.). New York: McGraw-Hill/Irwin.

Bokor, Z. (2010). Cost drivers in transport and logistics. Periodica Polytechnica Transportation Engineering, 38(1), 13-17.

Carneiro, D. M. (2015). Determinantes de Custos: uma proposta de sistematização. Dissertação (Mestrado em Controladoria e Contabilidade: Contabilidade) - Faculdade de Economia, Administração e Contabilidade, University of São Paulo, São Paulo, 2015.

Catânio, A. R. (2017). A Influência da Estratégia nos Determinantes de Custos e no Desempenho: um estudo no APL moveleiro de Arapongas/PR sob a perspectiva da teoria da contingência. Dissertação (Mestrado em Controladoria e Contabilidade: Contabilidade) Universidade Estadual de Maringá, Maringá, 2017.

Chea, A. (2011). Activity-Based Costing System in the Service Sector: A Strategic Approach for Enhancing Managerial Decision Making and Competitiveness. International Journal of Business and Management.

Cokins, G., \& Căpuşneanu, S. (2010). Cost Drivers. Evolution and Benefits. Theoretical and Applied Economics, 8(549), 7-16.

Cokins, Gary. (2002). Activity-based cost management: an executive's guide (Vol. 10). John Wiley \& Sons, Inc.

Collins, F., \& Werner, M. L. (1990). Improving Performance with Cost Drivers. Journal of Accountancy, 169(6), 131.

Cooper, R., \& Kaplan, R. S. (1988a). How Cost Accounting Systematically Distorts Product Costs. Management Accounting, 69(10), $20-27$.

Cooper, R., \& Kaplan, R. S. (1988b). Measure Costs Right: Make the Right Decision. Harvard Business Review, 66(5), 96-103.

Cooper, R., \& Kaplan, R. S. (1991). Profit Priorities from Activity-Based Costing. Harvard Business Review, 69, $130-135$.

Cooper, R., \& Kaplan, R. S. (1992). Activity-Based Systems: Measuring the Costs of Resource Usage. Accounting Horizons, 6, 1-13.

Cooper, R., \& Slagmulder, R. (1998). The scope of strategic cost management. Strategic Finance, 79, 16-18.

Costa, S. A. (2011). Análises de custos de concorrentes: um estudo dos determinantes de custos no setor de eletroeletrônicos. Dissertação (Mestrado em Controladoria e Contabilidade: Contabilidade) - Faculdade de Economia, Administração e Contabilidade, Universidade de São Paulo, São Paulo.

Costa, S. A. da, \& Carneiro, D. M. (2014). Determinantes de Custos: uma proposta de taxonomia e agrupamento. In: Congresso Brasileiro de Custos, 21; 2014. Natal.

Costa, S. A. da, \& Rocha, W. (2014). Determinantes de custos de concorrentes: identificação a partir de informações públicas. Revista de Gestao e Contabilidade Da UFPI, 1(1).

Datar, S. M., Kekre, S., Mukhopadhyay, T., \& Srinivasan, K. (1993). Simultaneous Estimation of Cost Drivers. The Accounting Review, 68(3), 602-614.

Diehl, C. A., Miotto, G. R., \& Souza, M. A. (2012). Análise da tecnologia das aeronaves como determinante de custos no setor de aviação comercial brasileiro. Revista Brasileira de Gestão de Negócios, 12(35), 191-207.

Dixon, R., \& Smith, D. (1993). Strategic management accounting. Omega, 21(6), 605-618.

El Kelety, I. A. E. M. A. (2006). Towards a conceptual framework for strategic cost management - The concept , objectives , and instruments -. Complexity.

Estermann, T., \& Claeys-Kulik, A.-L. (2013). Financially Sustainable Universities. Full Costing: Progress and Practice. EUA project. Brussels, Belgium: European University Association.

Foster, G., \& Gupta, M. (1990). Manufacturing overhead cost driver analysis. Journal of Accounting and Economics, 12(1-3), 309-337.

Geiger, D. R. (1999). Practical issues in cost driver selection for managerial costing systems. The Government Accountants Journal, 48(3), 32-39. 
Hansen, D. R., \& Mowen, M. M. (2003). Gestão de custos: contabilidade e controle. (R. B. Taylor, Trans.) (1st ed.). São Paulo: Pioneira Thompson Learning.

Hansen, D. R., \& Mowen, M. M. (2007). Managerial Accounting (8th ed.). South-Western College Pub.

Hansen, D. R., Mowen, M. M., \& Guan, L. (2007). Cost management: accounting and control (6th ed.). Mason, OH: South-Western College Pub.

Hoffjan, A., \& Kruse, H. (2006). Open Book Accounting in Supply Chains -- When and How is it Used in Practice? Cost Management, 20(6), 40-47.

Homburg, C. (2001). A note on optimal cost driver selection in ABC. Management Accounting Research, 12(2), $197-205$.

Horngren, C. T., Datar, S. M., \& Rajan, M. (2014). Cost accounting: a managerial emphasis (15th ed.). Pearson Education.

Kaplan, R. S. (2014). Improving value with TDABC. Healthcare Financial Management, 68(6), 76-83.

Kaplan, R. S., \& Cooper, R. (1998a). Cost \& Effect: Using Integrated Cost Systems to Drive Profitability and Performance. Boston: Harvard Business School Press.

Kaplan, R. S., \& Cooper, R. (1998b). Custo e desempenho: administre seus custos para ser mais competitivo. São Paulo: Futura.

Lebas, M. (1999). Which ABC? Accounting based on causality rather than activity-based costing. European Management Journal, 17(5), 501-511.

Lord, B. R. (1996). Strategic management accounting: the emperor's new clothes? Management Accounting Research, 7(3), 347-366.

Lyly-Yrjanâinen, J., Kulmala, H. I., \& Paranko, J. (2000). A Practical Activity-Based Costing Application in Logistics Busines. In In: 2nd Conference on New Directions in Management Accounting: Innovations in Practice and Research (p. 16). Brussels.

Mário, P. do C. (2013). A análise de cadeia de valor como ferramenta de criação de valor: um ensaio sobre a relação entre cost drivers e value drivers. In In: IX Convenção de Contabilidade do Rio Grande do Sul.

Miller, J. G., \& Vollmann, T. E. (1985). The hidden factory. Harvard Business Review, 63(5), 142-150.

Nakagawa, M. (1994). ABC: custeio baseado em atividades. São Paulo: Altas.

Noreen, E. (1991). Conditions under which activity-based cost systems provide relevant costs. Journal of Management Accounting Research, 3(3), 159-168.

Onwuegbuzie, A. J., \& Frels, R. (2016). Seven steps to a comprehensive literature review: A multimodal and cultural approach. Sage.

Panarella, P. J. M. (2010). Gestão e mensuração de custos: semelhanças e divergências entre a microeconomia e a contabilidade gerencial. Dissertação (Mestrado em Controladoria e Contabilidade: Contabilidade) - Faculdade de Economia, Administração e Contabilidade, Universidade de São Paulo, São Paulo, 2010.

Perčević, H., \& Lutilsky, I. D. (2008). Cost Allocation Accounting Methods Used in the Croatian Production Sector. South East European Journal of Economics and Business, 3(1), 49-57.

Pingxin, W., Fei, D., Dinghua, L., \& Lin, T. W. (2010). The Choice of Cost Drivers in Activity-Based Costing: Application at a Chinese Oil Well Cementing Company. International Journal of Management, 27(2), 367-380.

Porter, M. E. (1985). Competitive Advantage: Creating and sustaining superior performance. New York (Vol. 15). Free Press.

Porter, M. E. (1989). Vantagem competitiva: Criando e sustentando um desempenho superior. Rio de Janeiro: Elsevier.

Rocha, W. (1999). Contribuição ao estudo de um modelo conceitual de sistema de informação de gestão estratégica. Tese (Doutorado em Ciências Contabeis) - Faculdade de Economia, Administração e Contabilidade, Universidade de São Paulo, São Paulo, 1999.

Santos, F. B. (2014). Determinantes de custos na limpeza predial terceirizada: benchmarking em universidades federais. Dissertação (Mestrado em Controladoria e Contabilidade: Contabilidade) - Faculdade de Economia, Administração e Contabilidade, Universidade de São Paulo, São Paulo, 2014.

Schniederjans, M. J., \& Garvin, T. (1997). Using the analytic hierarchy process and multi-objective programming for the selection of cost drivers in activity-based costing. European Journal of Operational Research.

Severino, A. J. (2007). Metodologia do trabalho cientifico (23rd ed.). São Paulo: Cortez.

Shank, J. K. (1989). Strategic Cost Management: New Wine, or Just New Bottles? Journal of Management Accounting Rese$\operatorname{arch}, 1,47-65$.

Shank, J. K., \& Govindarajan, V. (1993). Strategic cost management: the new tool for competitive advantage. New York: The Free Press.

Shank, J. K., \& Govindarajan, V. (1997). A revolução dos custos: como reinventar e redefinir sua estratégia de custos para vencer em mercados crescentemente competitivos (2nd ed.). Rio de Janeiro: Campus.

Sheng, Y. . (2009). Research on selection methods of cost driver. Journal of Modern Accounting and Auditing, 5(9), 47-49.

Slavov, T. N. B. (2013). Gestão Estratégica de Custos: uma contribuição para a construção de sua estrutura conceitual. Tese (Doutorado em Controladoria e Contabilidade: Contabilidade) - Faculdade de Economia, Administração e Contabilidade, Universidade de São Paulo, São Paulo.

Slavov, T. N. B., \& Takahashi, M. (2010). Além do direcionamento de custos: a visão dos Determinantes de Custos - Cost Drivers - na Gestão Estratégica de Custos. In: Congresso Brasileiro de Custos, 18; 2010. 
Souza, B. C. de, \& Rocha, W. (2009). Gestão de custos interorganizacionais: ações coordernadas entre clientes e fornecedores para otimizar resultados. São Paulo: Altas.

Souza, M. A. De, \& Mezzomo, F. (2012). Determinantes de custos em empresas de pequeno porte do setor moveleiro: um estudo em empresas da serra gaúcha. Revista de Contabilidade e Controladoria, 4(1), 131-149.

Thiel, G. R., Rech, M., Souza, M. A., \& Machado, D. G. (2017). Determinantes de Custos: Análise Bibliométrica da Produção Científica em Periódicos Nacionais de Contabilidade, no período de 2000 a 2015. Revista Estudo \& Debate, 24(1).

Toompuu, K., \& Põlajeva, T. (2014). Theoretical Framework and an Overview of the Cost Drivers that are Applied in Universities for Allocating Indirect Costs. Procedia - Social and Behavioral Sciences, 110, 1014-1022.

Wrubel, F. (2009). Informações sobre gestão estatrégica de custos divulgadas por companhias abertas brasileiras. Universidade do Vale do Rio do Sinos, São Leopoldo. 